\title{
Detection of Railroad Networks in SAR Images
}

\author{
Şafak Altay Açar ${ }^{1}$ \\ Department of Computer Technologies \\ Yenice Vocational School, Karabük University \\ Karabük, Turkey
}

\author{
Şafak Bayır ${ }^{2}$ \\ Department of Computer Engineering \\ Faculty of Engineering, Karabük University \\ Karabük, Turkey
}

\begin{abstract}
In this study, a railroad networks detection method for synthetic aperture radar (SAR) images is proposed. Proposed method consists of three steps. Firstly, railroad segments are detected. An existing line detector is modified by describing some rules for this process. Then segments are connected by utilizing perceptual grouping. Finally, a new line analysis algorithm is applied to determine real parts of railroad networks. A software is developed to achieve and evaluate proposed method. Completeness and correctness values which are obtained after different steps are computed to evaluate proposed method. Two different TerraSAR- $X$ images are used in experiments and obtained results are discussed in detail.
\end{abstract}

Keywords-Remote sensing; synthetic aperture radar; railroad networks detection; perceptual grouping

\section{INTRODUCTION}

Synthetic aperture radar (SAR) is a system which can remote sensing and imaging. SAR could be set on satellites and aerial vehicles. SAR works effectively all day and night, regardless of all weather conditions [1]. These properties increase usability of SAR in civilian and military studies. Majority of passenger and freight transportations is done by roads and railroads. Also, location information of roads and railroads has strategic importance when a sensitive case occurs such as a civilian security. Because of these situations, studies which are about road and railroad detection have high importance but railroads are not taken into consideration as much as roads in studies.

There are many academic studies which extract roads from SAR images [2-13] but there are a few studies which determine railroads using SAR's qualities. Hellwich and Streck [14] present a study which searches visibility of linear structures like roads, rivers and railroads in SAR coherence data. Franceschetti et al. [15] propose an approach which extracts features from SAR raw data. They apply the proposed method to detect railroads. Pigeon et al. [16] present a research which extracts roads, railroads, rivers and energy transmission lines from SAR image. They use rules which are improved by human experts. Okman et al. [17] propose an approach for despeckling SAR images and present a semi-automated railroad detection algorithm to evaluate the performance of proposed despeckling method. A study is presented for polarimetric SAR image classification by Liu et al. [18]. They classify the terrain areas as road, railroad etc. Jin et al. [19] develop a constant false alarm line detector for polarimetric SAR images. They use Wilks' test statistics to develop the

This work was supported by the Scientific Research Coordination Unit of Ankara Yıldırım Beyazıt University as a preliminary research project - 631. detector. The detector determines bright and dark structures such as railroads and roads. In experimental study, they detect buildings and roads.

In addition to SAR imaging studies, many studies are made on railroad determination using various remote sensing and aerial images. Niu et al. [20] present an algorithm to extract linear features from remote sensing images. This algorithm which is based on beamlet transform and wedgelet decomposition is used for railroad detection. Pacifici et. al. [21] present a neural network study for urban area classification. They classify urban land-use as road, railroad, soil, building, tower, tree etc. using extracted textural features from satellite images. Beger et al. [22] propose an approach for railroad track and centre line detection from aerial images and airborne laser scanning data. They use a feature level data fusion to increase accuracy and completeness. Karaman et al. [23] present a fourier based study for railroad extraction. They develop a fourier based feature descriptor to detect railroad objects in aerial images.

Furthermore, there are various studies which use perceptual grouping. Some of them are about road extraction from SAR images $[5,8,11]$. Others use perceptual grouping in different areas. Hirogaki et al. [24] present a study which carries out complete three dimensional retrieval of dental cast shape. They use perceptual grouping to reconstruct whole shape. Qi et al. [25] propose an approach which draws sketch automatically. A single natural image is utilized for this process. Perceptual grouping is used to create contour segment groups in this approach. Wang et al. [26] present a research which extracts buildings from optical satellite images by utilizing distinctive image primitives. They use a graph search-based perceptual grouping to connect detected line segments. Qi et al. [27] propose a perceptual grouping framework that uses multiple Gestalt principles. They study to increase usefulness of image edges on various researches. A study is presented for segmentation of three dimensional outdoor scenes by Xu et al. [28]. They use hierarchical clustering structure and perceptual grouping laws.

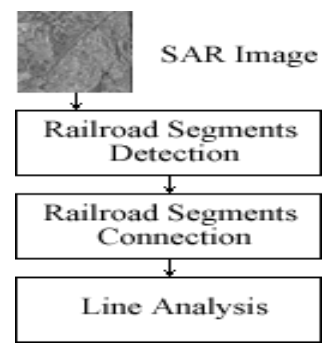

Fig. 1. Workflow of method. 
When the previous studies that we can obtain are taken into consideration, it is seen that there are not many studies on railroad detection on SAR images and also the studies are not carried out especially to detect railroads. In this study, which differs from the previous studies, railroad networks detection is achieved and evaluated individually. Improved method is fully automatic and extracts railroads from SAR images directly. We use cross-correlation line detector [2] to detect railroad segments. Then segments are connected by utilizing perceptual grouping. Finally, line analysis is applied to delete non-railroad pixels. Workflow of method is shown in Fig. 1.

The rest of the paper is organized as follows: Section II presents railroad segments detection. In section III, railroad segments connection is explained. Section IV presents line analysis process. In Sections V and VI, experimental results and conclusions are evaluated.

\section{RAILROAD SEGMENTS DETECTION}

In SAR images, railroads appear as bright structures. Furthermore, railroads are narrower than roads. According to these differences, detector model which is proposed in [2] is redesigned for railroad. These model is shown in Fig. 2. There are three regions in this model. Centre region is brighter than adjacent regions. Also, widths of regions are different. Some rules which are related to this model are described. Variables which are used in these rules are similar to the ones used in [29] for roads. Cross-correlation line detector [2] is performed for only pixels which provide rules. By means of these rules, areas which have high probability of being only railroads are investigated. Therefore, more accurate results are obtained and the time is saved. Table $\mathrm{I}$ reports rules. In Table $\mathrm{I}, \mathrm{p}(\mathrm{x}, \mathrm{y})$ denotes related pixel and $\mu_{1}, \mu_{2}$ and $\mu_{3}$ denote mean values of regions.

Firstly, 3x3 Gaussian filter is applied to smooth SAR image. Then, according to detector model, railroad segments are detected. Different region sizes are used for the model. There are two kinds of situations for detector model. Table II reports them. In Table II, $\ell$ denotes length of regions and $\mathrm{w}_{1}$, $\mathrm{w}_{2}, \mathrm{w}_{3}$ denote width of regions.

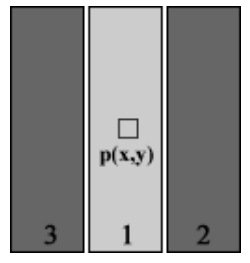

Fig. 2. Model of Railroad Detector.

TABLE I. DESCRIBED RULES

\begin{tabular}{|l|}
\hline colour ofp $(\mathrm{x}, \mathrm{y})>100$ \\
\hline$\mu_{1}>100$ \\
\hline$\mu_{1}-\mu_{2}>5$ \\
\hline$\mu_{1}-\mu_{3}>5$ \\
\hline
\end{tabular}

TABLE II. SIZE OF REGIONS

\begin{tabular}{|l|l|l|l|}
\hline$\ell($ pixel $)$ & $\mathrm{W}_{3}$ (pixel) & $\mathrm{W}_{1}($ pixel $)$ & $\mathrm{W}_{2}$ (pixel) \\
\hline 11 & 2 & 1 & 2 \\
\hline 11 & 2 & 2 & 2 \\
\hline
\end{tabular}

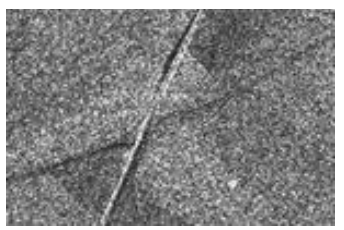

(a)

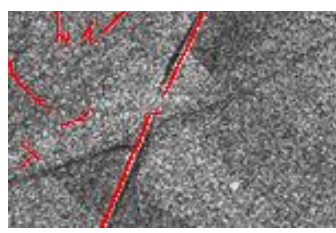

(b)
Fig. 3. Railroad Segments Detection Sample (a) Original Image (B) Detected Railroad Segments.

Detection process is applied for each situation, then results are combined. Eight different directions are tested and the best one is accepted as a result. Decision threshold is defined as 0.4 in the experiments. A railroad segments detection sample is shown in Fig. 3. Red regions denote detected railroad segments.

\section{RAILROAD SEGMENTS CONNECTION}

Detected railroad segments are connected by utilizing perceptual grouping so that railroad networks are generated. Proximity and cocurvilinearity which are denoted in [5] are used as perceptual grouping factor. They are shown in Fig. 4.

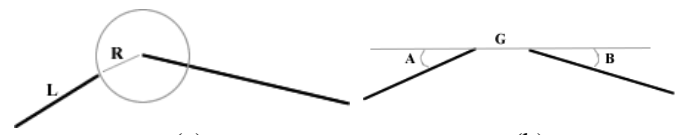

(a)

(b)

Fig. 4. Perceptual Grouping Factors (A) Proximity (B) Cocurvilinearity [5].

Proximity is formulated by (1). In the equation, $\mathrm{L}$ is the minimum length of two segments and $\mathrm{R}$ is the minimum distance between two segments at their endpoints. Cocurvilinearity is formulated by (2). In the equation, A and B are tangent angles of segments at joined endpoints. Coefficients $\alpha$ and $\beta$ control the deviation from collinearity and the sensitivity of $\mathrm{G}$ respectively [5]. In the experimental studies, we use them as $\alpha=10$ and $\beta=0.1$. Equations (1) and (2) are same as the ones used in $[5,8,11]$.

$P=\frac{L^{2}}{2 \pi R^{2}}$

$C=\frac{1}{\left(A^{2}+B^{2}\right)+(\alpha+\beta G)}$

Before connection process, segments whose sizes are equal or less than 20 pixels are deleted in order not to waste time with unnecessary segments. Then, segments which are longer than predefined threshold $\left(\mathrm{th}_{\mathrm{c}}\right)$ are determined. In the experiments, two different th $\mathrm{h}_{\mathrm{c}}$ values (30 and 100) are used and results are evaluated separately. Regions having $41 \times 41$ pixels which are around relevant segment's endpoints are searched. If there is any segment and tangent angles (A and B) which are less than $50^{\circ}$, then the value $\mathrm{V}$ defined in (3) is calculated. Segments which have the highest $\mathrm{V}$ values are grouped with each other. $\mathrm{P}$ and $\mathrm{C}$ values are multiplied by weighting factors as utilized in the studies $[5,8]$. In (3), $\gamma$ and $\theta$ are weighting factors. They are set to 0.5 and $10^{3}$ respectively in the experiments.

$V=\gamma P+\theta C$ 




(a)

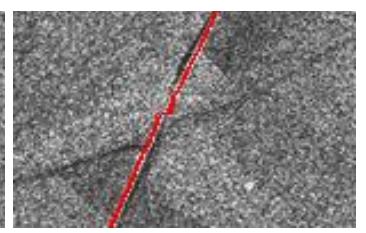

(b)
Fig. 5. Railroad Segments Connection Sample (A) Detected Railroad Segments (B) Image which is Obtained after Connection Process.

After connection process, segments whose sizes are equal or less than 100 pixels are deleted. A railroad segments connection sample is shown in Fig. 5. In Fig. 5(a), red regions denote detected railroad segments. Image which is obtained after connection process is shown in Fig. 5(b).

\section{LINE ANALYSIS}

A line analysis is applied to determine real parts of railroad networks. A new algorithm is proposed for this process.Adjacent pixels of relevant pixel which is a part of a detected railroad segment are searched. Kernel which is improved for searching is shown in Fig. 6. It consists of three regions as seen in Fig. 6. Red curve denotes a detected railroad segment and $\mathrm{p}_{\mathrm{r}}(\mathrm{x}, \mathrm{y})$ denotes pixel which is a part of detected railroad segment.

Kernel is placed on the $\mathrm{p}_{\mathrm{r}}(\mathrm{x}, \mathrm{y})$ for different directions and each time, numbers of detected pixels as railroad which are intersect with centre region and adjacent regions are calculated. If the total number of pixels which intersect with centre region are higher than predefined threshold $\left(\mathrm{th}_{1}\right)$ and if the total number of pixels which intersect with adjacent regions are less than predefined threshold $\left(\mathrm{th}_{2}\right), \mathrm{p}_{\mathrm{r}}(\mathrm{x}, \mathrm{y})$ remains as a part of segment. Ifthese conditions are not provided for any directions, $\mathrm{p}_{\mathrm{r}}(\mathrm{x}, \mathrm{y})$ is eliminated from the segment.

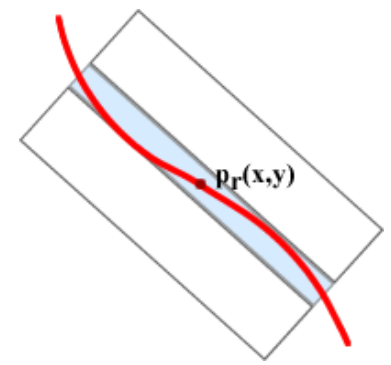

Fig. 6. Line Analysis Kernel.

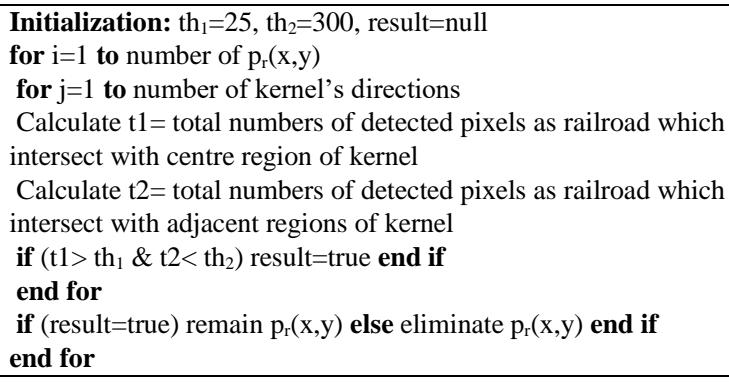

Fig. 7. Line Analysis Algorithm for Railroads.

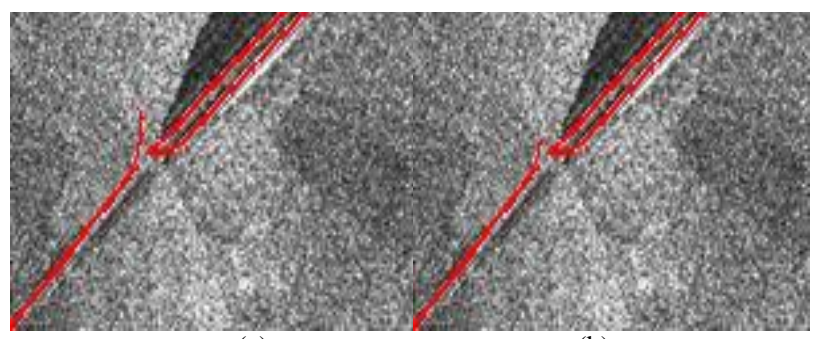

(a)

(b)

Fig. 8. Line Analysis Sample (A) Connected Railroad Segments (B) Image which is Obtained after Line Analysis Process.

This process is applied for all pixels which are parts of detected segments. In the experiments, $t_{1}$ and $t_{2}$ are used as 25 and 300 respectively. These thresholds are determined by experimental studies. Length of kernel, width of adjacent regions and width of centre region are 91, 11 and 2 pixels respectively. Width of centre region is determined according to maximum $\mathrm{w}_{1}$ of detector model which is explained in section II.

Line analysis algorithm for railroads is shown in Fig. 7 and a line analysis sample is shown in Fig. 8. In Fig. 8(a), red regions denote connected railroad segments. Image which is obtained after line analysis process is shown in Fig. 8(b).

\section{EXPERIMENTAL RESULTS}

A software was developed for realizing and testing our method. We applied method to two different SAR images (spotlight mode, multi look ground range, $\mathrm{HH}$ polarization and up to $2 \mathrm{~m}$ resolution). First image was acquired by TerraSAR-X over Polatlı (Ankara, Turkey). Second image was acquired by TerraSAR-X over Karaman (Turkey). These regions are selected since both of them include long railroads. Each of them covers a rural region of $10 \mathrm{~km} \mathrm{x} 10 \mathrm{~km}$. Images are resized, in other words, reduced in the ratio of $1 / 6$ (totally $1 / 36$ ) so first and second image become 2576 x 2299 pixels and 2553 x 2328 pixels respectively.

Sample results of images' railroad networks detection are shown in Fig. 9 and Fig. 10. In Fig. 9(b) and Fig. 10(b), red regions denote reference railroads. In Fig. 9(c) and Fig. 10(c), red regions denote detected railroads.

We compare real reference railroads with detected railroads pixel by pixel. Used formulas to calculate completeness and correctness values are defined in (4) and (5) respectively. These formulas are similar to the ones described in [30]. Reference pixels are determined by manually. A reference pixel is taken as matched reference pixel if there is a detected pixel in $3 \times 3$ pixels around and a detected pixel is taken as matched detected pixel if there is a reference pixel in $3 \times 3$ pixels around.

$$
\begin{aligned}
& \text { comp. }=\frac{\text { number_of_matched_reference_pixels } \times 100}{\text { number_of_reference_pixels }} \\
& \text { corr. }=\frac{\text { number_of_matched_detected_pixels } \times 100}{\text { number_of_detected_pixels }}
\end{aligned}
$$




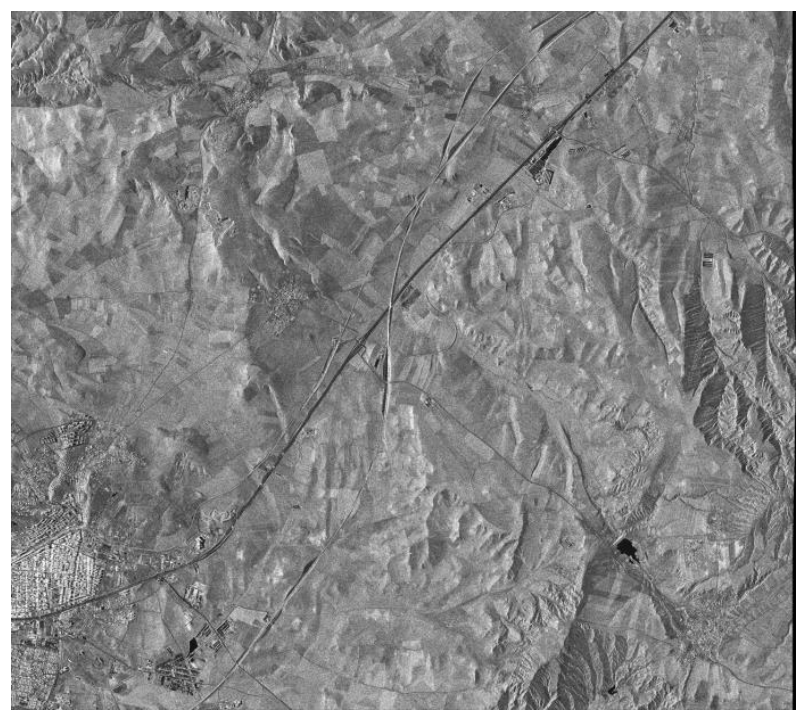

(a)

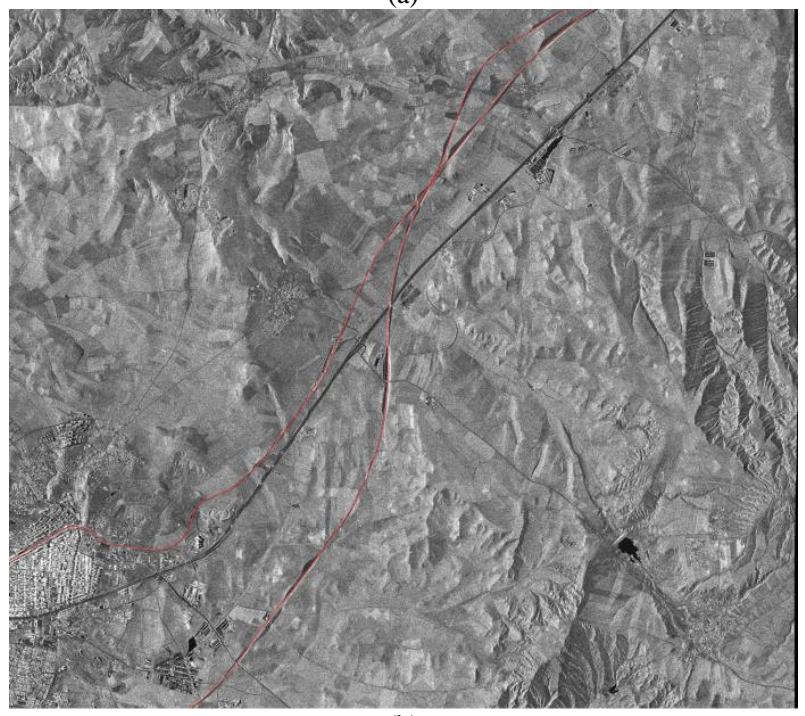

(b)

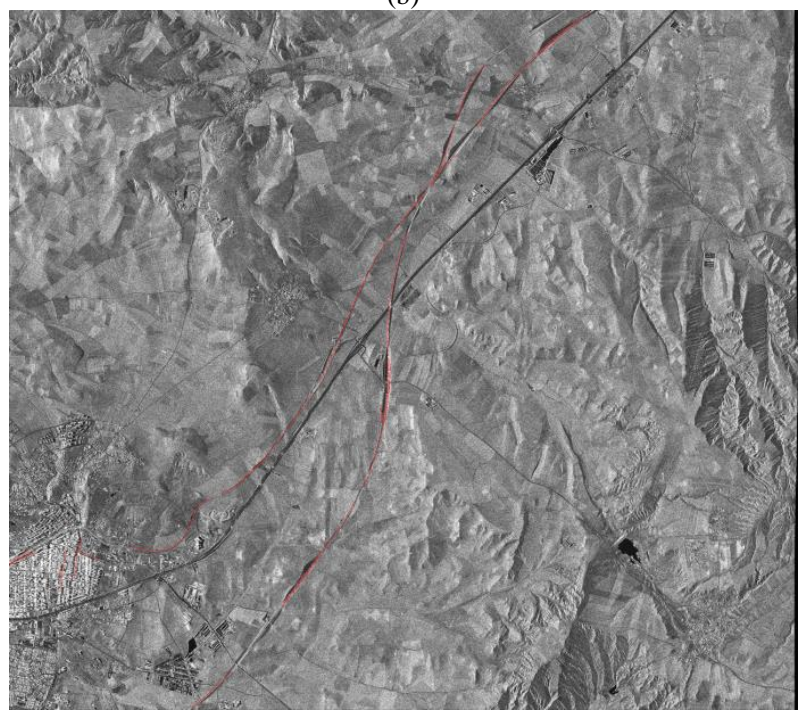

(c)

Fig. 9. First Image's Results (A) Original Image (B) Reference Railroads (C) Railroad Networks Detection $\left(\mathrm{th}_{\mathrm{c}}=30\right.$ Completeness Value: 68.26 Correctness Value: 76.11)

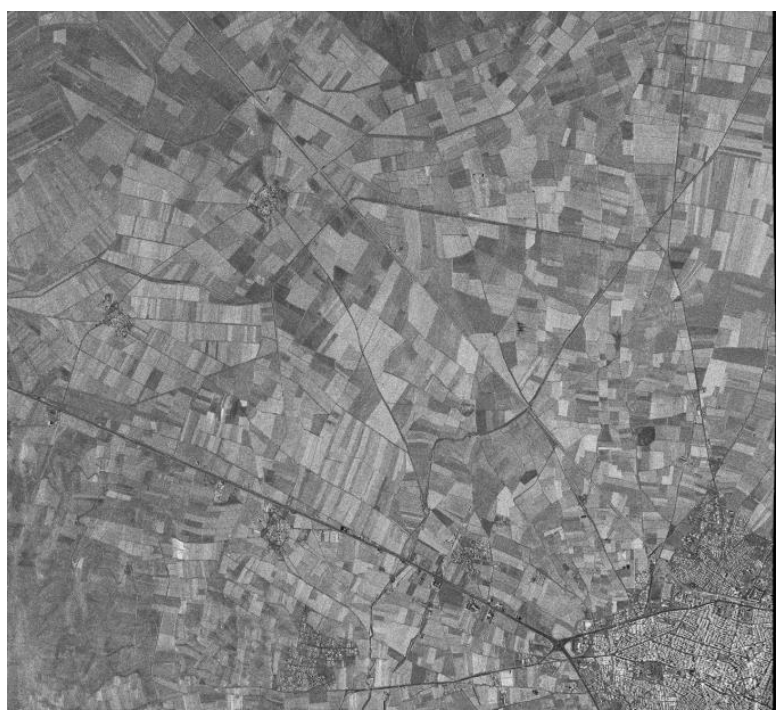

(a)
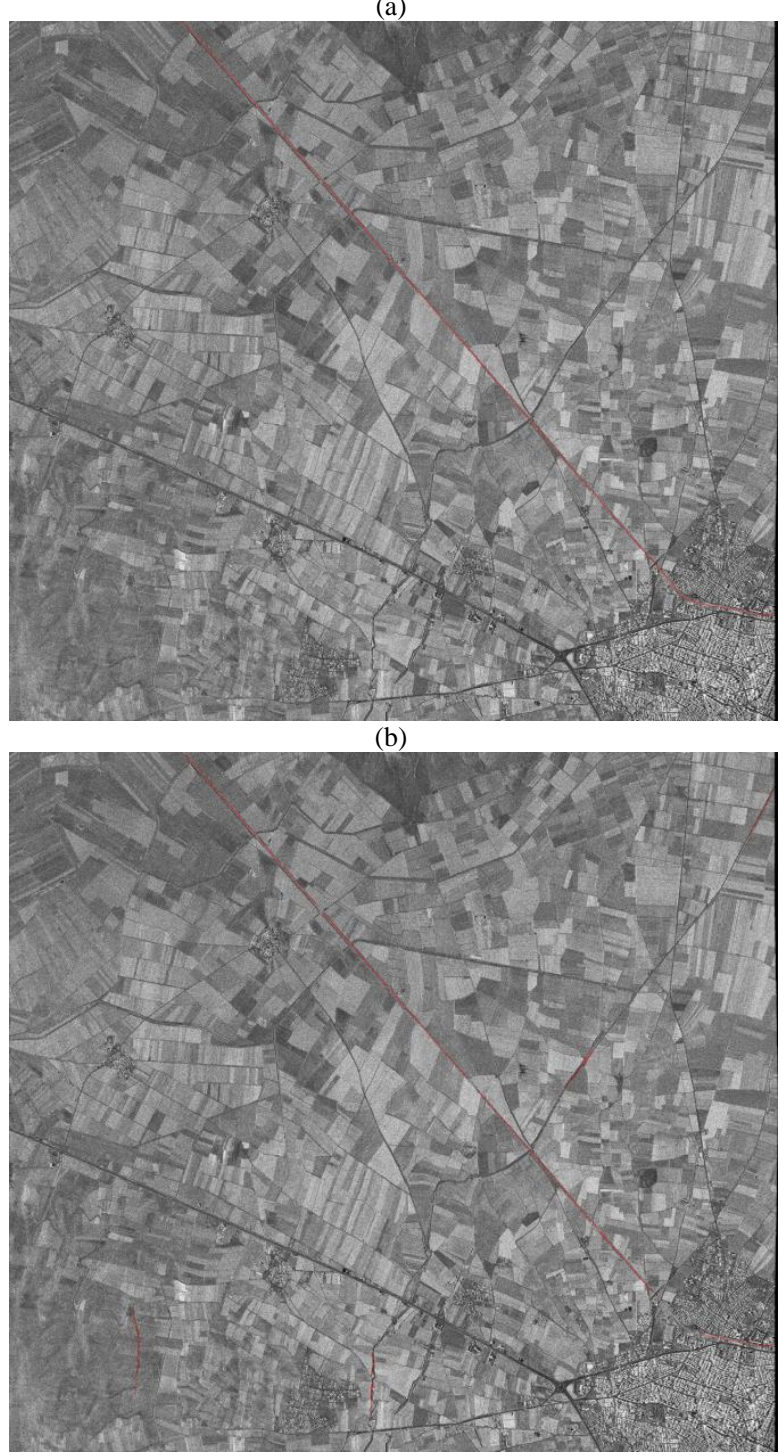

(c)

Fig. 10. Second Image's Results (A) Original Image (B) Reference Railroads (C) Railroad Networks Detection ( th $_{\mathrm{c}}=30$ Completeness Value: 88.84 Correctness Value: 74.07). 
TABLE III. EVALUATION OF RAILROAD NETWORKS DETECTION FOR THE FIRST IMAGE $\left(\mathrm{TH}_{\mathrm{C}}=30\right)$

\begin{tabular}{|l|l|l|l|l|l|l|}
\hline \multirow{2}{*}{$\delta$} & \multicolumn{2}{|l|}{ After Connection } & \multicolumn{2}{l|}{ After Line Analysis } & \multicolumn{2}{l|}{ After Cleaning } \\
\cline { 2 - 7 } & Comp. & Corr. & Comp. & Corr. & Comp. & Corr. \\
\hline 100 & 79.79 & 30.22 & 79.20 & 34.32 & 70.94 & 72.87 \\
\hline 300 & 69.96 & 70.89 & 69.48 & 72.31 & 68.26 & 76.11 \\
\hline 500 & 58.64 & 73.17 & 58.11 & 74.20 & 55.94 & 78.16 \\
\hline
\end{tabular}

TABLE IV. EVALUATION OF RAILROAD NETWORKS DETECTION FOR THE SECOND IMAGE $\left(\mathrm{TH}_{\mathrm{C}}=30\right)$

\begin{tabular}{|l|l|l|l|l|l|l|}
\hline \multirow{2}{*}{$\delta$} & \multicolumn{2}{|l|}{ After Connection } & \multicolumn{2}{l|}{ After Line Analysis } & \multicolumn{2}{l|}{ After Cleaning } \\
\cline { 2 - 7 } & Comp. & Corr. & Comp. & Corr. & Comp. & Corr. \\
\hline 100 & 94.02 & 22.24 & 93.59 & 25.06 & 93.59 & 58.16 \\
\hline 300 & 89.26 & 61.40 & 88.84 & 65.43 & 88.84 & 74.07 \\
\hline 500 & 71.35 & 93.99 & 71.04 & 95.13 & 71.04 & 97.23 \\
\hline
\end{tabular}

Evaluations of railroad networks detection are given in Tables III, IV, V and VI. After segment detection and connection processes, segments which have small sizes are deleted. The threshold relevant to deleted segments sizes is denoted as $\delta$ in tables. After connection process, line analysis and cleaning are applied. In cleaning process, segments whose sizes are equal or less than 200 pixels are deleted. Two different $t_{c}$ values (30 and 100) are used and results are evaluated separately. The results of $t_{c}=30$ are given in Tables III and IV and the results of $t_{c}=100$ are given in Tables V and VI.

Results of each processes for the first image are given in Tables III and V. Results of each processes for the second image are given in Tables IV and VI.

According to results, assessments occur as follows:

- When $\delta$ value increases, completeness decreases but correctness increases.

- After line analysis, completeness decreases but correctness increases.

- For the first image, after cleaning, completeness decreases but correctness increases. When $\delta$ is 100 pixel, these decrement and increment are more than other $\delta$ values.

- For the second image, after cleaning, completeness does not change but correctness increases. When $\delta$ is 100 pixel, this increment is more than other $\delta$ values.

TABLE V. EVALUATION OF RAILROAD NETWORKS DETECTION FOR THE FIRST IMAGE $\left(\mathrm{TH}_{\mathrm{C}}=100\right)$

\begin{tabular}{|l|l|l|l|l|l|l|}
\hline \multirow{2}{*}{$\delta$} & \multicolumn{2}{|l|}{ After Connection } & \multicolumn{2}{l|}{ After Line Analysis } & \multicolumn{2}{l|}{ After Cleaning } \\
\cline { 2 - 7 } & Comp. & Corr. & Comp. & Corr. & Comp. & Corr. \\
\hline 100 & 66.92 & 65.09 & 66.15 & 67.12 & 55.66 & 91.02 \\
\hline 300 & 59.70 & 86.62 & 58.99 & 87.50 & 55.66 & 91.07 \\
\hline 500 & 47.40 & 85.24 & 46.82 & 86.60 & 46.51 & 90.67 \\
\hline
\end{tabular}

TABLE VI. EVALUATION OF RAILROAD NETWORKS DETECTION FOR THE SECOND IMAGE $\left(\mathrm{TH}_{\mathrm{C}}=100\right)$

\begin{tabular}{|c|c|c|c|c|c|c|}
\hline \multirow{2}{*}{$\delta$} & \multicolumn{2}{|c|}{ After Connection } & \multicolumn{2}{c|}{ After Line Analysis } & \multicolumn{2}{c|}{ After Cleaning } \\
\cline { 2 - 7 } & Comp. & Corr. & Comp. & Corr. & Comp. & Corr. \\
\hline 100 & 86.07 & 51.64 & 85.42 & 54.53 & 80.49 & 80.29 \\
\hline 300 & 80.91 & 87.72 & 80.49 & 88.42 & 80.49 & 88.76 \\
\hline 500 & 80.91 & 97.64 & 80.49 & 97.89 & 80.49 & 97.89 \\
\hline
\end{tabular}

According to results, assessments occur as follows:

- When $\delta$ value increases, completeness decreases or does not change but correctness usually increases.

- After line analysis, completeness decreases but correctness increases.

- For the first image, after cleaning, completeness decreases but correctness increases. When $\delta$ is 100 pixel, these decrement and increment are more than other $\delta$ values.

- For the second image, after cleaning, completeness usually does not change but correctness increases or does not change. When $\delta$ is 100 pixel, this increment is more than other $\delta$ values.

When we compare the results obtained when th $h_{c}$ value is equal to 100 with the results obtained when th $\mathrm{c}_{\mathrm{c}}$ value is equal to 30 , it is seen that the completeness value is generally lower, on the other hand, the correctness value is higher.

\section{CONCLUSION}

This study presents an efficient railroad networks detection method for SAR images. The method is tested by utilizing two SAR images. Completeness and correctness values are calculated for each one and obtained results are evaluated in detail. Proposed method can be modified to detect any continuous curvilinear structures.

A useful method will be developed in order to apply to reduce false detections so that correctness value will increases by not affecting completeness value. Furthermore, proposed method will be improved to work by using less constants.

\section{REFERENCES}

[1] A. Moreira, P. Prats-Iraola, M. Younis, G. Krieger, I. Hajnsek, and K. P. Papathanassiou, "A tutorial on synthetic aperture radar," IEEE Geosci. Remote Sensing Mag., vol. 1, no. 1, pp. 6-43, Mar. 2013.

[2] F. Tupin, H. Maitre, J. F. Mangin, J. M. Nicolas, and E. Pechersky, "Detection of linear features in SAR images: application to road network extraction," IEEE Trans. Geosci. Remote Sens., vol. 36, no. 2, pp. 434453, Mar. 1998.

[3] J. Chanussot, G. Mauris, and P. Lambert, "Fuzzy fusion techniques for linear features detection in multitemporal SAR images," IEEE Trans. Geosci. Remote Sens., vol. 37, no. 3, pp. 1292-1305, May 1999.

[4] F. Dell'Acqua and P. Gamba, "Detection of urban structures in SAR images by robust fuzzy clustering algorithms: the example of street tracking," IEEE Trans. Geosci. Remote Sens., vol. 39, no. 10, pp. 22872297, Oct. 2001.

[5] B. Jeon, J. Jang, and K. Hong, "Road detection in spaceborne SAR images using a genetic algorithm," IEEE Trans. Geosci. Remote Sens., vol. 40, no. 1, pp. 22-29, Jan. 2002.

[6] P. Gamba, F. Dell'Acqua, and G. Lisini, "Improving urban road extraction in high-resolution images exploiting directional filtering, 
perceptual grouping, and simple topological concepts," IEEE Geosci. Remote Sens. Lett., vol. 3, no. 3, pp. 387-391, Jul. 2006.

[7] D. Borghys, Y. Yvinec, C. Perneel, A. Pizurica, and W. Philips, "Supervised feature-based classification of multi-channel SAR images," Pattern Recognition Lett., vol. 27, no. 4, pp. 252-258, Mar. 2006.

[8] Y. Guo, Z. Bai, Y. Li, and Y. Liu., "Genetic algorithm and region growing based road detection in SAR images," in Proc. Third Int. Conference on Natural Computation, Haikou, 2007, pp. 330-334.

[9] H. Chaabouni-Chouayakh and M. Datcu, "Coarse-to-fine approach for urban area interpretation using TerraSAR-X data," IEEE Geosci. Remote Sens. Lett., vol. 7, no. 1, pp. 78-82, Jan. 2010.

[10] J. Cheng, W. Ding, X. Ku, and J. Sun, "Road extraction from highresolution SAR images via automatic local detecting and human-guided global tracking," Int. Journal of Antennas and Propagation, Nov. 2012.

[11] C. He, Z. Liao, F. Yang, X. Deng, and M. Liao, "Road extraction from SAR imagery based on multiscale geometric analysis of detector responses," IEEE J. Sel. Topics Appl. Earth Observ. Remote Sens., vol. 5, no. 5, pp. 1373-1382, Oct. 2012.

[12] M. Saati, J. Amini, and M. Maboudi, "A method for automatic road extraction of high resolution SAR imagery," Journal of the Indian Society of Remote Sensing, vol. 43, no. 4, pp. 697-707, Dec. 2015.

[13] M. Jiang, Z. Miao, P. Gamba, and B. Yong, "Application of multitemporal inSAR coveriance and information fusion to robust road extraction," IEEE Trans. Geosci. Remote Sens., vol. 55, no. 6, pp. 36113622, Jun. 2017.

[14] O. Hellwich and C. Streck, "Linear structures in SAR coherence data," in Proc. Int. Geosci. and Remote Sens. Symposium, Lincoln, USA, 1996, vol. 1, pp. 330-332.

[15] G. Franceschettia, A. Iodice, and M. Tesauroa, "From image processing to feature processing," Signal Processing, vol. 60, no. 1, pp. 51-63, Jul. 1997.

[16] L. Pigeon, B. Solaiman, K. P. B. Thomson, B. Moulin, and T. Toutin, "Human-experts rules modeling for linear planimetric features extraction in a remotely sensed images data fusion context," in Proc. IEEE Int. Geosci. and Remote Sens. Symposium, Hamburg, Germany, 1999, vol. 5, pp. 2369 - 2371.

[17] O. E. Okman, F. Nar, and C. Demirkesen, "Feature preserving sar despeckling and its parallel implementation with application to railway detection," in Proc. 9th European Conference on Synthetic Aperture Radar, Nuremberg, Germany, 2012, pp. 151-154.

[18] F. Liu, J. Shia, L. Jiao, H. Liu, S. Yang, J. Wu, H. Hao, and J. Yuan, "Hierarchical semantic model and scattering mechanism based PolSAR image classification," Pattern Recognition, vol. 59, pp. 325-342, Nov. 2016.
[19] R. Jin, W. Zhou, J. Yin, and J. Yang, "CFAR line detector for polarimetric SAR images using Wilks' test statistic," IEEE Geosci. Remote Sens. Lett., vol. 13, no. 5, pp. 711-715, May. 2016.

[20] R. Niu, X. Mei, L. Zhang, and P. Li, "Linear features extraction from remote sensing image based on wedgelet decomposition," in Proc. Fourth Int. Conference on Image and Graphics, Sichuan, China, 2007, pp. $508-512$.

[21] F. Pacifici, M. Chini, and W. J. Emery, "A neural network approach using multi-scale textural metrics from very high-resolution panchromatic imagery for urban land-use classification," Remote Sensing of Environment, vol. 113, no. 6, pp. 1276-1292, Jun. 2009.

[22] R. Beger, C. Gedrange, R. Hect, and M. Neubert, "Data fusion of extremely high resolution aerial imagery and LiDAR data for automated railroad centre line reconstruction," ISPRS Journal of Photogrammetry and Remote Sens., vol. 66, no. 6, pp. S40-S51, Dec. 2011.

[23] E. Karaman, U. Çinar, E. Gedik, Y. Yardımcı, and U. Halıcı, "Fourier based feature descriptors for railroad extraction from aerial images," in Proc. IEEE Int. Geosci. and Remote Sens. Symposium, Munich, Germany, 2012, pp. 6013 - 6015.

[24] Y. Hirogaki, T. Sohmura, H. Satoh, J. Takahashi, and K. Takata, "Complete 3-D reconstruction of dental cast shape using perceptual grouping," IEEE Trans. Medical Imaging, vol. 20, no. 10, pp. 10931101, Oct. 2001.

[25] Y. Qi, J. Guo, Y. Li, H. Zhang, T. Xiang, and Y. Song, "Sketching by perceptual grouping," in Proc. 20th IEEE Int. Conference on Image Processing, Melbourne, Australia, 2013, pp. 270-274.

[26] J. Wang, X. Yang, X. Qin, X. Ye, and Q. Qin, "An efficient approach for automatic rectangular building extraction from very high resolution optical satellite imagery," IEEE Geosci. Remote Sens. Lett., vol. 12, no. 3, pp. 487-491, Mar. 2015.

[27] Y. Qi, Y. Song, T. Xiang, H. Zhang, T. Hospedales, Y. Li, and J. Guo, "Making better use of edges via perceptual grouping," in Proc. IEEE Conference on Computer Vision and Pattern Recognition, Boston, USA, 2015, pp. 1856-1865.

[28] Y. Xu, S. Tuttas, and U. Stilla, "Segmentation of 3D outdoor scenes using hierarchical clustering structure and perceptual grouping laws," in Proc. 9th IAPR Workshop on Pattern Recognition in Remote Sensing, Cancun, Mexico, 2016.

[29] Ş. Altay Açar and Ş. Bayır, "Effect of threshold values used for road segments detection in SAR images on road network generation," International Journal of Advanced Computer Science and Applications, vol. 8, no. 5, pp.287-291, May. 2017.

[30] C. Heipke, H. Mayer, C. Wiedemann, and O. Jamet, "Evaluation of automatic road extraction," Int. Archives of Photogrammetry and Remote Sens., vol. 32, pp. 47-56, 1997. 